\title{
Medicinal plant remedies for dermatological problems
}

\author{
M. C. Sidhu, Sweta Thakur* \\ Department of Botany, Panjab University, Chandigarh, India
}

Received: 21.01.2017

Accepted: 27.02.2017

Published: 28.02.2017

*Address for correspondence:

Sweta Thakur, Department of Botany, Panjab University, Chandigarh, India.

E-mail: shbthkr@gmail.com

\begin{abstract}
This study has been undertaken to document the medicinal plants used to take care of various dermatological problems - such as cuts, burns, itching, abscesses, allergies etc. in district Mandi of Himachal Pradesh (India). A total of 1000 respondents were contacted to gather this information using a semi-structured questionnaire. This study has highlighted the use of 168 species belonging to 148 genera and 67 families for the purpose under consideration. Most of the plants were herbs followed by shrubs, trees, climber, and liana. Leaves were the most frequently used plant part. Medicinal preparations include paste, juice, powder etc. It indicates that the respondents have sound knowledge about the medicinal plants and their utility.
\end{abstract}

KEY WORDS: Ethnobotany, Himachal Pradesh, Mandi, medicinal potential, plant species, skin problems

\section{INTRODUCTION}

Ethnobotanical knowledge related to medicinal plants may add new discoveries to the world of medicines. Plantbased medicines are considered cost-effective and safe as compared to synthetic drugs. Furthermore, people gain economic benefits by collecting medicinal plants from the forests and selling them in market (Lazarou et al., 1998; White et al., 1999; Uniyal and Shiva, 2005). This has elevated the growth of herbal medicine industries. Many plant species are yet to be explored, especially for their various medicinal activities. Most of the information is still restricted to some traditional healers or passed on to next generation by word of mouth or even lost (Akharaiyi and Boboye, 2010; Yirga, 2010). Therefore, it is of immense importance to conserve this precious knowledge before it vanished. The documentation and identification of plants are very important, not only for their utilization but conservation also (Muthu et al., 2006). The skin is an effective protective system. It protects the body from various infections by inhibiting the entry of pathogens. However, some of the pathogens and environmental conditions may cause problems such as eczema, leucoderma, wounds, and itching (Govindasamy and Arulpriya, 2013; Suresh et al., 2012). Previously, ethnobotanical studies, related to dermatological problems have also been conducted in different countries on the use of plants to treat various skin diseases (Joshi and Joshi,
2007; Njoroge and Bussmann, 2007; Abbasi et al., 2010; Wet et al., 2013; Sirsawat et al., 2016).

\section{MATERIALS AND METHODS}

\section{Study Area}

This study has been undertaken in district Mandi of Himachal Pradesh. The district lies between $31^{\circ} 13^{\prime} 50^{\prime \prime}$ and $32^{\circ} 04^{\prime} 30^{\prime \prime} \mathrm{N}$ latitude and between $76^{\circ} 37^{\prime} 20^{\prime \prime}$ and $77^{\circ} 23^{\prime} 15^{\prime \prime}$ E longitude. The total geographical area of the district is $3951 \mathrm{~km}^{2}$ which is $7.1 \%$ of area of state. The average annual rainfall is about $1331.50 \mathrm{~mm}$ (Central Ground Water Board, 2013). The prevailing variety of climatic conditions has enriched the study area with floristic diversity. The district is comprised of 10 tehsils. Ethnobotanical survey for this study was conducted using method followed by Sidhu and Thakur (2015). The respondents were interviewed for the use of plants species for various dermatological problems such as burns, cuts, pimples, wounds etc.

Plant species were photographed during the flowering or fruiting seasons using Samsung PL50 digital camera preferably in their natural habitats. The plants which were difficult to identify from the photographs were collected as a specimen. Later, they were identified with the help of specimens present in a herbarium, Department of Botany, Panjab University, Chandigarh; Herbal Garden 
and Herbarium, Research Institute in Indian System of Medicine, Joginder Nagar. The voucher specimens (of 76 species) were pressed, dried, labeled, and deposited in the herbarium of Department of Botany, Panjab University, Chandigarh.

\section{RESULTS AND DISCUSSION}

A total of 168 plant species from 148 genera and 67 families were used for the treatment of various skin problems. The families, botanical names, local names, habit, source, method of preparation, and uses are given in Table 1. The families - such as Lamiaceae, Asteraceae, and Fabaceae - have contributed 13, 12, and 10 members, respectively (Table 2). The main source was wild species
(127) followed by cultivated (33) species. Four species occur both in wild as well as cultivated state whereas the products of remaining four were purchased from the market. The herbs (72) were dominating among the reported plant species (Figure 1).

All the recorded plant species (168) were useful in the treatment of more than 28 skin related problems including abscesses, burns, itching, pimple, skin rashes, urticaria, warts etc. Maximum number of species (58) were used to treat abscesses followed by cuts (57), burns (38), fungal infection on toes (19), irritation/ itching (17), and so on (Figure 2). Ajuga parviflora and Aloe vera were used to remove dark scars from burns. Nine remedies were available for the treatment of

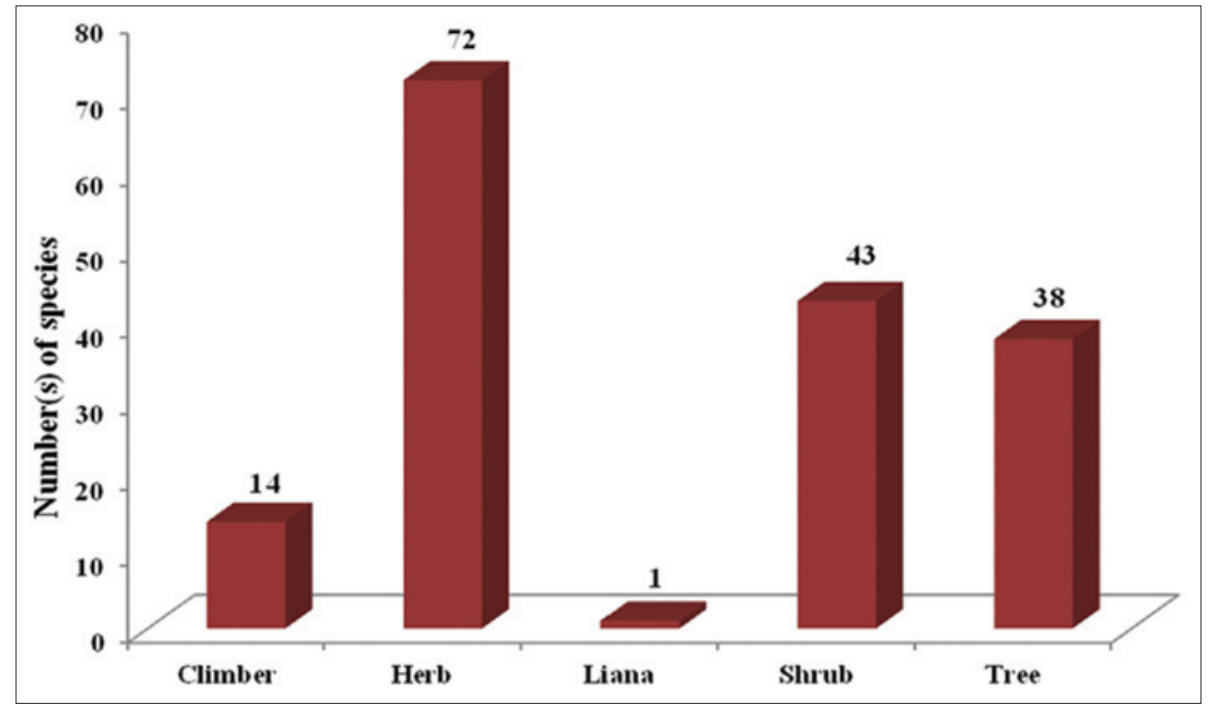

Figure 1: Habits of medicinal plants

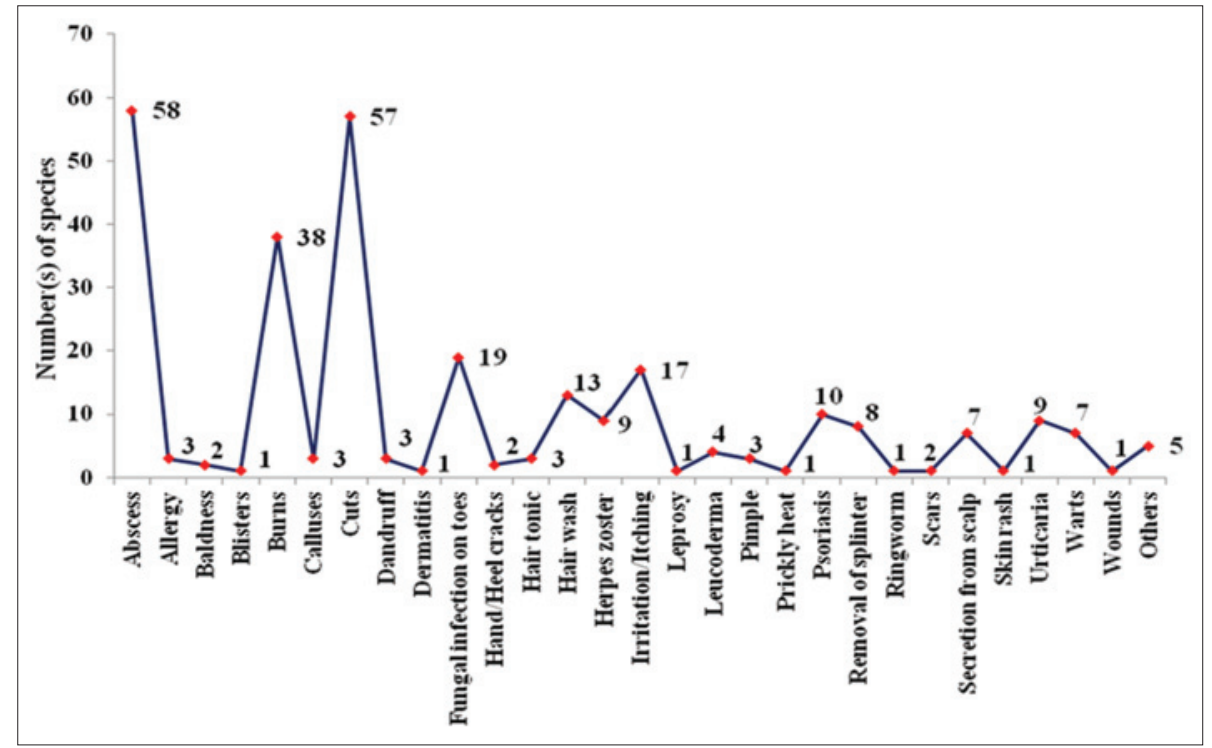

Figure 2: Number of plant species to cure various skin problems 
Table 1: Plant species for dermatological problems

\begin{tabular}{|c|c|c|c|}
\hline Families/botanical name of plants & Local name & $\mathrm{Ht} / \mathrm{Sc}$ & Part used and preparation (application/s) \\
\hline \multicolumn{4}{|l|}{ Acanthaceae } \\
\hline D. bupleuroides Nees & Suandi & H/W & Leaf paste (abscesses) \\
\hline J. adhatoda L. & Basuti & $\mathrm{S} / \mathrm{W}$ & $\begin{array}{l}\text { Roots and leaves paste (cuts) } \\
\text { Warm leaves (abscesses) }\end{array}$ \\
\hline \multicolumn{4}{|l|}{ Amaranthaceae } \\
\hline A. aspera L. & Puthkanda & H/W & Leaf juice (cuts) \\
\hline A. bidentata Blume & Umbal koori & $\mathrm{H} / \mathrm{W}$ & Root paste (abscesses) \\
\hline C. tomentosa (Roth) Moq. & Koori & S/W & Paste of whole plant (cuts) \\
\hline D. amaranthoides (Lam.) Merr. & Bhirange & S/W & Leaf paste (abscesses) \\
\hline \multicolumn{4}{|l|}{ Amaryllidaceae } \\
\hline A. cepa L. & Pyaz & $\mathrm{H} / \mathrm{C}$ & Pounded bulb is tied (abscesses) \\
\hline Z. carinata Herb. & Yaadu & H/W & $\begin{array}{l}\text { Paste is made by crushing small part of bulbs with seeds of } S \text {. mukorossi } \\
\text { and } V \text {. mungo and mixing with gel of } A \text {. vera (abscesses) }\end{array}$ \\
\hline \multicolumn{4}{|l|}{ Anacardiaceae } \\
\hline \multirow[t]{2}{*}{ M. indica L. } & Aamb & $\mathrm{T} / \mathrm{C}$ & Fruit stalk exudates (calluses) \\
\hline & & & $\begin{array}{l}\text { Dry fruit pieces are fried in mustard oil to make paste (skin burns) } \\
\text { Kernel paste (cuts) }\end{array}$ \\
\hline \multicolumn{4}{|l|}{ Apiaceae } \\
\hline \multirow[t]{4}{*}{ C. asiatica (L.) Urb. } & Brahmi & $\mathrm{H} / \mathrm{W}$ & Leaf juice (cuts) \\
\hline & & & Leaf paste (abscesses, itching) \\
\hline & & & Leaf paste (burns, urticaria) \\
\hline & & & Leaves are given with black pepper and butter (leucoderma) \\
\hline C. sativum L. & Been & $\mathrm{H} / \mathrm{C}$ & Leaf paste (cuts) \\
\hline \multicolumn{4}{|l|}{ Apocynaceae } \\
\hline C. procera (Aiton) Dryand. & Aak & S/W & $\begin{array}{l}\text { Leaf paste (skin burns) } \\
\text { Latex (warts) }\end{array}$ \\
\hline C. spinarum L. & Garna & S/W & Latex (calluses, warts, remove splinters, skin blisters) \\
\hline C. roseus (L.) G. Don & Sadabahar & $\mathrm{S} / \mathrm{C}$ & Leaf paste is applied with black pepper (psoriasis) \\
\hline C. dubia (Burm. f.) M. R. Almeida & Khurma & L/W & Kernel paste (Skin diseases) \\
\hline \multicolumn{4}{|l|}{ Araceae } \\
\hline A. tortuosum (Wall.) Schott & Jhangas & $\mathrm{H} / \mathrm{W}$ & $\begin{array}{l}\text { Tuber paste with black pepper (abscesses) } \\
\text { Leaf paste (leucoderma) }\end{array}$ \\
\hline C. esculenta (L.) Schott & Kachalu, beju & $\mathrm{H} / \mathrm{C}$ & $\begin{array}{l}\text { Warm leaves (fungal infection of toes) } \\
\text { Water exudates (warts) }\end{array}$ \\
\hline \multicolumn{4}{|l|}{ Arecaceae } \\
\hline C. nucifera L. & Nariyal & $\mathrm{T} / \mathrm{M}$ & Oil (skin burns) \\
\hline \multicolumn{4}{|l|}{ Asparagaceae } \\
\hline \multirow[t]{2}{*}{ A. racemosus Willd. } & Sainsor booti & $\mathrm{Cl} / \mathrm{W}$ & Leaf paste (skin burns) \\
\hline & & & $\begin{array}{l}\text { Crushed fruits are mixed in water to make froth and applied on } \\
\text { scalp (wash hair) }\end{array}$ \\
\hline D. indica (Roxb.) Jessop & Ban-pyaz & $\mathrm{H} / \mathrm{W}$ & Bulb paste (abscesses) \\
\hline \multicolumn{4}{|l|}{ Asteraceae } \\
\hline $\begin{array}{l}\text { A. adenophora (Spreng.) R. M. King and } \\
\text { H. Rob. }\end{array}$ & Baslo ghaas & S/W & Leaf paste (cuts) \\
\hline A. conyzoides (L.) L. & Ujaru & H/W & Leaf juice (cuts) \\
\hline A. nilagirica (C. B. Clarke) Pamp. & Kubsha & S/W & Leaf juice (cuts) \\
\hline A. vulgaris $L$. & Charmara & S/W & Leaf juice (cuts) \\
\hline B. anthelmintica (L.) Moench & Brahmjiri & $\mathrm{H} / \mathrm{W} / \mathrm{C}$ & $\begin{array}{l}\text { Crushed seeds are eaten with black pepper and honey (abscesses, itching) } \\
\text { Seed paste is applied with mustard oil (itching) }\end{array}$ \\
\hline C. minima (L.) A. Braun and Asch. & Nk-chikku & H/W & Whole plant paste (abscesses) \\
\hline E. prostrata (L.) L. & Bhringraj & H/W & $\begin{array}{l}\text { Crushed seeds are warmed in mustard oil and applied on scalp (hair } \\
\text { tonic) }\end{array}$ \\
\hline G. gossypina (Royle) Beauverd & Baacha & H/W & Leaf paste (cuts) \\
\hline T. erecta L. & Sartaj & $\mathrm{S} / \mathrm{C}$ & Inflorescence paste (skin burns) \\
\hline T. patula L. & Sutajri, golu, dolari & $\mathrm{S} / \mathrm{C}$ & $\begin{array}{l}\text { Leaf juice (cuts) } \\
\text { Leaf paste (pimples, skin burns) }\end{array}$ \\
\hline T. campylodes G. E. Haglund & Dudhali & H/W & Latex (cuts) \\
\hline T. procumbens (L.) L. & Phulnu & H/W & $\begin{array}{l}\text { Crushed plant is taken as such or made into tablets with black } \\
\text { pepper (abscesses, herpes zoster) }\end{array}$ \\
\hline \multicolumn{4}{|l|}{ Balsaminaceae } \\
\hline I. balsamina L. & Dioond & H/W & Leaf paste (fungal infection of toes) \\
\hline \multicolumn{4}{|l|}{ Berberidaceae } \\
\hline B. aristata DC. & Kashmal & S/W & Cold decoction of roots is used dermally (cuts, itching) \\
\hline Bignoniaceae & & & \\
\hline O. indicum (L.) Kurz. & Arlu & $\mathrm{T} / \mathrm{W}$ & Crushed seeds are warmed in oil (dermatitis) \\
\hline & & & Bark powder mixed with sesame oil (psoriasis) \\
\hline
\end{tabular}


Table 1: (Continued)

\begin{tabular}{|c|c|c|c|}
\hline Families/botanical name of plants & Local name & $\mathrm{Ht} / \mathrm{Sc}$ & Part used and preparation (application/s) \\
\hline \multicolumn{4}{|l|}{ Bombacaceae } \\
\hline \multirow[t]{2}{*}{ B. ceiba L. } & Semul & $\mathrm{T} / \mathrm{W}$ & Leaf paste (cuts) \\
\hline & & & Thorns are scrubbed to form paste (abscesses) \\
\hline \multicolumn{4}{|l|}{ Boraginaceae } \\
\hline \multirow[t]{3}{*}{ C. zeylanicum (Vahl) Brand } & Koori & H/W & Root paste (abscesses) \\
\hline & & & Leaf juice (cuts) \\
\hline & & & Leaf paste (fungal infection of toes) \\
\hline \multicolumn{4}{|l|}{ Brassicaceae } \\
\hline \multirow[t]{2}{*}{ B. rapa L. } & Saron & $\mathrm{H} / \mathrm{C}$ & Seed oil (itching) \\
\hline & & & Oil with turmeric powder (skin burns) \\
\hline E. vesicaria (L.) Cav. & Taara-mira & $\mathrm{H} / \mathrm{C}$ & Crushed seeds are added to warm oil (itching) \\
\hline \multicolumn{4}{|l|}{ Cannabaceae } \\
\hline C. sativa L. & $\begin{array}{l}\text { Bhaang, bhangolu, } \\
\text { vijaya }\end{array}$ & $\mathrm{H} / \mathrm{W}$ & Leaves paste (urticaria, skin irritation caused due to U. dioica) \\
\hline \multicolumn{4}{|l|}{ Caprifoliaceae } \\
\hline V. jatamansi Jones & Nihani & $\mathrm{H} / \mathrm{W}$ & Roots and leaves paste (cuts) \\
\hline \multicolumn{4}{|l|}{ Caricaceae } \\
\hline C. papaya L. & Papita & $\mathrm{T} / \mathrm{C}$ & Latex (psoriasis) \\
\hline \multicolumn{4}{|l|}{ Combretaceae } \\
\hline T. arjuna (Roxb. ex DC.) Wight and Arn. & Arjun & $T / C$ & Leaf paste; bark powder mixed with clarified butter (skin burns) \\
\hline T. chebula Retz. & Harar & $\mathrm{T} / \mathrm{W}$ & Fruit paste (fungal infection of toes) \\
\hline \multicolumn{4}{|l|}{ Convolvulaceae } \\
\hline \multirow[t]{3}{*}{ C. reflexa Roxb. } & Akashbel & $\mathrm{Cl} / \mathrm{W}$ & Plant juice (warts) \\
\hline & & & Plant paste (herpes zoster, urticaria) \\
\hline & & & Plant mixed in mustard oil (hair tonic) \\
\hline I. nil (L.) Roth & Ghaundani & $\mathrm{Cl} / \mathrm{W}$ & Seeds are pounded and dissolved in water (wash hair) \\
\hline \multicolumn{4}{|l|}{ Crassulaceae } \\
\hline \multirow[t]{2}{*}{ B. pinnatum (Lam.) Oken } & Patharchat, lusgadu & $\mathrm{H} / \mathrm{C}$ & Leaf juice (cuts) \\
\hline & & & Leaves are smeared with warm mustard oil (abscesses) \\
\hline S. glaucophyllum R. T. Clausen & Ludru & H/W & Leaf paste (burns, cuts, abscesses) \\
\hline \multicolumn{4}{|l|}{ Cucurbitaceae } \\
\hline C. sativus L. & Kakdi & $\mathrm{Cl} / \mathrm{C}$ & Fruit juice (skin burns) \\
\hline \multirow[t]{2}{*}{ C. sativus var. hardwickii (Royle) Gabaev } & Fafanu & $\mathrm{Cl} / \mathrm{W}$ & Fruits paste (cuts) \\
\hline & & & Warm fruit is applied with jaggery (abscesses) \\
\hline L. siceraria (Molina) Standl. & Ghiya & $\mathrm{Cl} / \mathrm{C}$ & Fruit peel (skin burns) \\
\hline \multicolumn{4}{|l|}{ Dioscoreaceae } \\
\hline D. deltoidea Wall. ex Griseb. & Shingli-mingli & $\mathrm{Cl} / \mathrm{W}$ & $\begin{array}{l}\text { Dry rhizomes are pounded, dissolved in water and froth is applied on } \\
\text { scalp (wash hair) }\end{array}$ \\
\hline \multicolumn{4}{|r|}{ 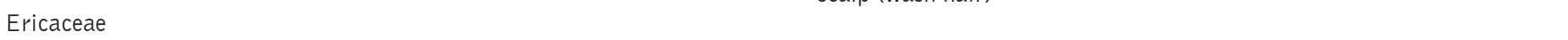 } \\
\hline L. ovalifolia (Wall.) Drude & Bheral & T/W & $\begin{array}{l}\text { Young twigs are crushed with young twigs of } P \text {. pashia and mixed in } \\
\text { mustard oil (itching) }\end{array}$ \\
\hline R. campanulatum D. Don. & Shargar & S/W & Leaf paste (urticaria) \\
\hline Euphorbiaceae & & & \\
\hline E. helioscopia L. & Dudhali & $\mathrm{H} / \mathrm{W}$ & $\begin{array}{l}\text { Stem paste (secretion from scalp) } \\
\text { Latex (cuts) }\end{array}$ \\
\hline E. heterophylla L. & Dudhali & H/W & Latex (cuts) \\
\hline E. hirta L. & Dudhali & H/W & Leaf paste (abscesses) \\
\hline & & & Latex (cuts, hand cracks) \\
\hline E. royleana Boiss. & Chhunha & S/W & $\begin{array}{l}\text { Small pieces of stem are fried in mustard oil till amount of oil reduced to } \\
1 / 10^{\text {th }} \text { of the original (itching, ringworm) }\end{array}$ \\
\hline & & & Latex (cuts, abscesses, remove splinter) \\
\hline F. insignis Royle & Dudhla, balodhar & $\mathrm{T} / \mathrm{W}$ & Latex, for short time (psoriasis, warts) \\
\hline J. curcas L. & Japhlota & S/W & $\begin{array}{l}\text { Crushed seeds are dissolved in water to form froth which is applied on } \\
\text { scalp (wash hair) }\end{array}$ \\
\hline & & & Latex (warts, fungal infection of toes, cuts) \\
\hline M. philippensis (Lam.) Mull. Arg. & Kamahl & $\mathrm{T} / \mathrm{W}$ & Leaf juice (itching (caused by fruits of $M$. pruriens) \\
\hline & & & $\begin{array}{l}\text { Glandular hairs (wounds formed by infection due to spine, skin or scalp } \\
\text { in itching problem) }\end{array}$ \\
\hline R. communis L. & Airn & S/W & Leaves are rubbed over hands and feet (burning sensation) \\
\hline & & & Seed paste (heel cracks) \\
\hline Fabaceae & & & \\
\hline A. precatorius L. & Chadainu & $\mathrm{Cl} / \mathrm{W}$ & Seed paste (abscesses, leucoderma) \\
\hline A. gageana Craib & Darnghodi, bagharne & S/W & Leaf paste (cuts) \\
\hline & & & Leaf powder is mixed with water (skin burns) \\
\hline B. variegata $\mathrm{L}$. & Karalein & T/W & Juice of twigs (skin burns) \\
\hline
\end{tabular}


Table 1: (Continued)

\begin{tabular}{|c|c|c|c|}
\hline Families/botanical name of plants & Local name & $\mathrm{Ht} / \mathrm{Sc}$ & Part used and preparation (application/s) \\
\hline B. monosperma (Lam.) Taub. & Palah & $\mathrm{S} / \mathrm{C}$ & $\begin{array}{l}\text { Flower paste is made by crushing with flowers of } \\
\text { H. rosa-sinensis (baldness) }\end{array}$ \\
\hline C. fistula L. & Aali & $\mathrm{T} / \mathrm{W}$ & Young leaves paste (fungal infection of toes) \\
\hline C. corylifolium (L.) Medik. & Babchi & $\mathrm{H} / \mathrm{C}$ & Leaf paste (leucoderma) \\
\hline I. heterantha Brandis & Kaathi & S/W & Leaf paste (fungal infection of toes) \\
\hline L. culinaris Medik. & Massar & $\mathrm{H} / \mathrm{C}$ & Paste of roasted seeds (skin burns, secretion from scalp) \\
\hline S. tora (L.) Roxb. & Reli & $\mathrm{H} / \mathrm{W}$ & Crushed seeds are applied with mustard oil (abscesses) \\
\hline V. mungo (L.) Hepper & Maah & $\mathrm{H} / \mathrm{C}$ & $\begin{array}{l}\text { Small amount of bulb of } Z \text {. carinata is crushed with seeds of } \\
S \text {. mukorossi and } V \text {. mungo and mixed with } A \text {. vera gel to form } \\
\text { paste (abscesses) }\end{array}$ \\
\hline \multicolumn{4}{|l|}{ Fagaceae } \\
\hline Q. incana Bartram & Baan & T/W & Ash of twigs (wash hair) \\
\hline \multicolumn{4}{|l|}{ Hypericaceae } \\
\hline $\begin{array}{l}\text { H. uralum Buch.-Ham. ex D. Don } \\
\text { Juglandaceae }\end{array}$ & Khradu & S/W & Leaf paste (cuts) \\
\hline J. regia L. & Akhrot, khod & $\mathrm{T} / \mathrm{W} / \mathrm{C}$ & Paste of fruit covering (fungal infection of toes) \\
\hline A. parviflora Benth. & Neelkanthi & $\mathrm{H} / \mathrm{W}$ & $\begin{array}{l}\text { Leaf juice (skin burns, removes dark scars, itching due to allergic } \\
\text { reactions) } \\
\text { Leaf paste (herpes zoster, abscesses, fungal infection of toes) } \\
\text { Leaves crushed in mustard oil (remove dandruff) } \\
\text { Whole plant paste (cuts) }\end{array}$ \\
\hline C. tomentosa (L.) L. & Dushti & S/W & Juice of young leaves (cuts) \\
\hline C. umbrosum (M. Bieb.) Kuntze & Barua & $\mathrm{H} / \mathrm{W}$ & Leaf paste (cuts) \\
\hline C. oppositifolia Sm. & Gadoosa & S/W & $\begin{array}{l}\text { Leaf juice (cuts) } \\
\text { Leaf paste (urticaria) }\end{array}$ \\
\hline I. wightii (Benth.) H. Hara & Kngaara & $\mathrm{S} / \mathrm{W}$ & Leaf paste (fungal infection of toes) \\
\hline L. lanata Benth. & Dhurlu ghaas & $\mathrm{H} / \mathrm{W}$ & $\begin{array}{l}\text { Paste is made by crushing its leaves with whole plant of Peperomia } \\
\text { tetraphylla and Ajuga parviflora (abscesses) }\end{array}$ \\
\hline $\begin{array}{l}\text { M. biflora (Buch.-Ham. ex D. Don) } \\
\text { Benth. }\end{array}$ & Marua & $\mathrm{H} / \mathrm{W}$ & Leaf paste (wounds) \\
\hline P. mollis (Aiton) Spreng. & Banbhabri & S/W & Leaf juice (skin burns, cuts) \\
\hline P. benghalensis (Burm. f.) Kuntze & $\begin{array}{l}\text { Kaali bansuti, } \\
\text { bhrmayara }\end{array}$ & S/W & $\begin{array}{l}\text { Leaf paste (cuts, fungal infection of toes) } \\
\text { Leaf paste (psoriasis) }\end{array}$ \\
\hline P. mollissima Roth & Bakar & T/W & $\begin{array}{l}\text { Leaf paste (psoriasis) } \\
\text { Leaves chutney is eaten (itching) }\end{array}$ \\
\hline R. cinerea (D. Don) Baill. & $\begin{array}{l}\text { Kadkoi, kdkhre, } \\
\text { kadwo, itsri }\end{array}$ & S/W & $\begin{array}{l}\text { Leaf paste (abscesses, itching) } \\
\text { Paste is made by crushing its leaves with leaves of } C \text {. asiatica and } \\
\text { A. parviflora (secretion from scalp) }\end{array}$ \\
\hline S. scandens D. Don & Chhichad & S/W & Leaf paste (fungal infection of toes) \\
\hline V. negundo L. & Sura, bnah & S/W & $\begin{array}{l}\text { Leaf juice (cuts) } \\
\text { Leaf paste (fungal infection of toes) }\end{array}$ \\
\hline \multicolumn{4}{|l|}{ Lauraceae } \\
\hline $\begin{array}{l}\text { C. tamala (Buch.-Ham.) T. Nees \& } \\
\text { Eberm. }\end{array}$ & Gudpatraj & T/W & Leaf decoction (dandruff) \\
\hline \multicolumn{4}{|l|}{ Linaceae } \\
\hline L. usitatissimum L. & Alsi & $\mathrm{H} / \mathrm{C}$ & $\begin{array}{l}\text { Ripened fruit of } C \text {. annuum is fried in its oil and pounded into } \\
\text { paste (cuts) }\end{array}$ \\
\hline R. indica Dumort. & Piyein-re-phool & H/W & Leaf and stem paste (cuts) \\
\hline \multicolumn{4}{|l|}{ Lythraceae } \\
\hline L. inermis L. & Mehndi & $\mathrm{T} / \mathrm{M}$ & Leaf paste (skin burns) \\
\hline P. granatum L. & Daru & S/W & Pericarp paste (fungal infection of toes) \\
\hline \multicolumn{4}{|l|}{ Malvaceae } \\
\hline G. optiva J. R. Drumm. ex Burret & Byul & T/W & $\begin{array}{l}\text { Young twig paste (cuts) } \\
\text { Young twigs are crushed and dissolved in water which is applied on } \\
\text { scalp (wash hair) }\end{array}$ \\
\hline H. rosa-sinensis L. & Gurhal & $\mathrm{S} / \mathrm{C}$ & $\begin{array}{l}\text { Flower paste is made by crushing with flowers of } \\
\text { B. monosperma (baldness) }\end{array}$ \\
\hline S. cordifolia L. & Daridein & $\mathrm{H} / \mathrm{W}$ & $\begin{array}{l}\text { Leaf juice (cuts) } \\
\text { Leaf paste (abscesses) }\end{array}$ \\
\hline \multicolumn{4}{|l|}{ Melanthiaceae } \\
\hline T. govanianum Wall. ex D. Don & Naag chatri & H/W & Root paste (cuts) \\
\hline A. indica A. Juss. & Neem & $\mathrm{T} / \mathrm{C}$ & $\begin{array}{l}\text { Leaf paste (fungal infection of toes, skin burns, secretion from scalp in } \\
\text { children) }\end{array}$ \\
\hline M. azedarach L. & Darek & T/W & Leaf extract is mixed with oil (hair tonic) \\
\hline
\end{tabular}


Table 1: (Continued)

\begin{tabular}{|c|c|c|c|}
\hline Families/botanical name of plants & Local name & $\mathrm{Ht} / \mathrm{Sc}$ & Part used and preparation (application/s) \\
\hline \multicolumn{4}{|l|}{ Menispermaceae } \\
\hline \multirow[t]{4}{*}{ C. pareira L. } & Patindu & & Root and leaves paste (abscesses) \\
\hline & & & Leaves are smeared with warm mustard oil (abscesses) \\
\hline & & & Whole plant paste (skin burns) \\
\hline & & & Paste of fresh leaves (cuts) \\
\hline C. laurifolius DC. & Parora & $\mathrm{S} / \mathrm{W}$ & Leaves and bark paste is mixed with black pepper (secretion from scalp) \\
\hline S. glabra (Roxb.) Miers & Bish khapra & $\mathrm{Cl} / \mathrm{W}$ & Tuber paste (abscesses) \\
\hline \multicolumn{4}{|l|}{ Moraceae } \\
\hline F. auriculata Lour. & Tryambla, debra & $\mathrm{T} / \mathrm{W}$ & Latex (cuts, remove splinter) \\
\hline F. benghalensis L. & Bar & T/W/C & $\begin{array}{l}\text { Decoction of crushed prop roots (wash hair) } \\
\text { Latex (cuts) }\end{array}$ \\
\hline F. carica L. & Khasra & $\mathrm{T} / \mathrm{W}$ & Latex (remove splinter, calluses) \\
\hline F. palmata Forssk. & Faigra & $\mathrm{T} / \mathrm{W}$ & Latex (cuts, itching, remove splinter) \\
\hline F. racemosa L. & Umraya & T/W & $\begin{array}{l}\text { Leaf juice (skin burns) } \\
\text { Latex (warts) }\end{array}$ \\
\hline F. religiosa L. & Peepal & T/W/C & $\begin{array}{l}\text { Paste of bark is applied with jaggery (abscesses) } \\
\text { Latex (leprosy) }\end{array}$ \\
\hline M. serrata Roxb. & Cheemu & $\mathrm{T} / \mathrm{W}$ & Resin (cuts) \\
\hline \multicolumn{4}{|l|}{ Musaceae } \\
\hline M. paradisiaca L. & Kela & $\mathrm{H} / \mathrm{C}$ & Leaf juice (skin burns) \\
\hline \multicolumn{4}{|l|}{ Myrtaceae } \\
\hline S. aromaticum (L.) Merr. \& L. M. Perry & Laung & $\mathrm{T} / \mathrm{M}$ & Paste flower bud (abscesses) \\
\hline \multicolumn{4}{|l|}{ Nyctaginaceae } \\
\hline M. jalapa L. & $\begin{array}{l}\text { Gulabaans, } \\
\text { daini-phool }\end{array}$ & S/W & Root paste (abscesses) \\
\hline \multicolumn{4}{|l|}{ Oleaceae } \\
\hline J. mesnyi Hance & Chameli & $\mathrm{Cl} / \mathrm{C}$ & Leaf paste (cuts) \\
\hline \multicolumn{4}{|l|}{ Orchidaceae } \\
\hline \multirow[t]{2}{*}{ A. multiflora Roxb. } & Bhangru & $\mathrm{H} / \mathrm{W}$ & Leaf juice (cuts) \\
\hline & & & Leaf juice is mixed with clarified butter and applied (skin burns) \\
\hline \multicolumn{4}{|l|}{ Oxalidaceae } \\
\hline \multirow[t]{2}{*}{ O. corniculata L. } & Malori & H/W & Whole plant juice (remove splinter) \\
\hline & & & Whole plant paste (abscesses, skin burns, prickly heat) \\
\hline \multicolumn{4}{|l|}{ Pedaliaceae } \\
\hline S. indicum L. & Til & $\mathrm{S} / \mathrm{C}$ & Pounded leaves are dissolved in water (wash hair) \\
\hline \multicolumn{4}{|l|}{ Phyllanthaceae } \\
\hline \multirow[t]{2}{*}{ P. emblica L. } & Amla & T/W & Leaf paste (fungal infection of toes) \\
\hline & & & Fruit paste (skin burns) \\
\hline \multirow{2}{*}{\multicolumn{4}{|c|}{ Piperaceae }} \\
\hline & & & \\
\hline \multicolumn{2}{|l|}{ P. tetraphylla (G. Forst.) Hook. and Arn. } & H/W & $\begin{array}{l}\text { Paste is made by crushing whole plant with leaves of } L \text {. lanata and } \\
\text { A. parviflora (abscesses) }\end{array}$ \\
\hline \multirow[t]{2}{*}{ P. nigrum L. } & Kali mirch & $\mathrm{Cl} / \mathrm{M}$ & Crushed fruits is applied with mustard oil (itching) \\
\hline & & & Fruit powder is given with clarified butter (urticaria) \\
\hline Plantaginaceae & & & \\
\hline P. depressa Willd. & Chasha & H/W & Leaves are smeared with warm mustard oil (abscesses) \\
\hline Plumbaginaceae & & & \\
\hline P. zeylanica L. & Chitra & S/W & Root paste (applied for very short time) (itching) \\
\hline Poaceae & & & \\
\hline C. dactylon (L.) Pers. & Doob & H/W & Plant juice (skin burns, cuts) \\
\hline D. falcatum (Nees) Keng f. & $\begin{array}{l}\text { Dhadhanj, nigaal, } \\
\text { gohra }\end{array}$ & H/W & $\begin{array}{l}\text { Leaves are boiled with leaves of } P \text {. mollissima and extract is used to take } \\
\text { bath (urticaria) }\end{array}$ \\
\hline E. coracana (L.) Gaertn. & Kodra, mandal & $\mathrm{H} / \mathrm{C}$ & Seed paste (herpes zoster) \\
\hline T. aestivum $\mathrm{L}$. & Kanak & $\mathrm{H} / \mathrm{C}$ & Wheat flour is applied dermally (skin burns) \\
\hline & & & $\begin{array}{l}\text { Wheat flour is fried to make "Halwa." It is cooled and applied } \\
\text { dermally (remove splinter) }\end{array}$ \\
\hline & & & Wheat germ oil (psoriasis) \\
\hline Z. mays L. & Chhalli & $\mathrm{H} / \mathrm{C}$ & $\begin{array}{l}\text { Water is added to the stem ash to obtain lye which is applied on } \\
\text { scalp (wash hairs) }\end{array}$ \\
\hline Polygonaceae & & & \\
\hline P. amplexicaulis (D. Don) Ronse Decr. & Dora & H/W & Root paste (abscesses) \\
\hline P. capitata (Buch.-Ham. ex D. Don) & Chasha & $\mathrm{H} / \mathrm{W}$ & Leaf powder (cuts) \\
\hline H. Gross & & & \\
\hline
\end{tabular}


Table 1: (Continued)

\begin{tabular}{|c|c|c|c|}
\hline Families/botanical name of plants & Local name & $\mathrm{Ht} / \mathrm{Sc}$ & Part used and preparation (application/s) \\
\hline R. nepalensis Spreng. & Albar, Marli & $\mathrm{H} / \mathrm{W}$ & $\begin{array}{l}\text { Root paste (abscesses, fungal infection) } \\
\text { Leaves are folded, warmed and kept on infected portion (remove splinter) } \\
\text { Leaves are warmed along with leaves of } N \text {. tabacum and kept (abscesses) }\end{array}$ \\
\hline \multicolumn{4}{|l|}{ Ranunculaceae } \\
\hline R. acris L. & Meenkli, Jakri & $\mathrm{H} / \mathrm{W}$ & $\begin{array}{l}\text { Paste of roots and leaves is applied after } 3 \text { days of injury (cuts, bone } \\
\text { fracture) }\end{array}$ \\
\hline $\begin{array}{l}\text { R. arvensis L. } \\
\text { Rosaceae }\end{array}$ & Jaldar & $\mathrm{H} / \mathrm{W}$ & Whole plant paste, for very short time (psoriasis) \\
\hline $\begin{array}{l}\text { Rosaceae } \\
\text { F. vesca L. }\end{array}$ & Bhui akha, laal aakhe & $\mathrm{H} / \mathrm{W}$ & $\begin{array}{l}\text { Paste of whole plant with black pepper }\{\text { abscesses (on forehead) } \\
\text { Whole plant paste (skin burns) }\end{array}$ \\
\hline P. supina L. & Oolti kuri & H/W & Root bark paste (abscesses) \\
\hline P. utilis Royle & Bhekhal & S/W & Root ash (abscesses) \\
\hline P. cerasoides Buch.-Ham. ex D. Don & Pajja & $\mathrm{T} / \mathrm{W}$ & Flowers paste mixed with curd and used (skin burns) \\
\hline P. pashia Buch.-Ham. ex D. Don & Kainth, shegal & $\mathrm{T} / \mathrm{W}$ & Paste of young twigs and fresh leaves (fungal infection of toes) \\
\hline R. ellipticus Sm. & Aakhe, heer & $\mathrm{S} / \mathrm{W}$ & $\begin{array}{l}\text { Root paste (skin burns) } \\
\text { Leaf paste (cuts, fungal infection of toes) }\end{array}$ \\
\hline \multicolumn{4}{|l|}{ Rubiaceae } \\
\hline C. spinosa (Thunb.) Tirveng. & Raada & S/W & $\begin{array}{l}\text { Fruits paste (skin rashes, pimples) } \\
\text { Fruits are crushed, dissolved in water to obtain froth which is used (wash } \\
\text { hair) }\end{array}$ \\
\hline $\begin{array}{l}\text { G. aparine L. } \\
\text { H. tetrasperma (Wall. ex Roxb.) T. Yamaz }\end{array}$ & Chirmitti, tushusho & $\begin{array}{l}\mathrm{H} / \mathrm{W} \\
\mathrm{S} / \mathrm{W}\end{array}$ & $\begin{array}{l}\text { Paste of aerial parts (abscesses, cuts, herpes zoster) } \\
\text { Leaf paste (herpes zoster) }\end{array}$ \\
\hline \multicolumn{4}{|l|}{ Rutaceae } \\
\hline C. limon (L.) Osbeck & Nimbu & $\mathrm{T} / \mathrm{C}$ & $\begin{array}{l}\text { Fruit juice is applied in infected area and left for } 2-3 \text { hours. It is } \\
\text { washed with water and butter is applied. The process is repeated for } \\
7 \text { days (psoriasis) }\end{array}$ \\
\hline C. pseudolimon Tanaka & Khatta & $\mathrm{T} / \mathrm{C}$ & $\begin{array}{l}\text { Fruit juice (Skin burns) } \\
\text { Warm fruit (Abscesses) }\end{array}$ \\
\hline \multicolumn{4}{|l|}{ Salicaceae } \\
\hline F. indica (Burm. f.) Merr. & Kangu & T/W & $\begin{array}{l}\text { Bark ash is applied with mustard oil (abscesses) } \\
\text { Thorn is scrubbed and paste is applied (abscesses) }\end{array}$ \\
\hline S. babylonica L. & Majnu & T/W & $\begin{array}{l}\text { Crushed young twigs are dissolved in water to form froth which is } \\
\text { applied on scalp (wash hair) }\end{array}$ \\
\hline \multicolumn{4}{|l|}{ Sapindaceae } \\
\hline A. indica (Wall. ex Cambess.) Hook. & Kanor & T/W & $\begin{array}{l}\text { Fruits are crushed and dissolved in water (wash hair) } \\
\text { Bark paste (abscesses) }\end{array}$ \\
\hline D. viscosa (L.) Jacq. & Mehandru & S/W & $\begin{array}{l}\text { Wet twigs are heated to collect sap which is used dermally (skin } \\
\text { problems) } \\
\text { Leaf powder and paste (skin burns) }\end{array}$ \\
\hline S. mukorossi Gaertn. & Dodae, ritha & T/W & Fruits are crushed and dissolved in water (wash hair) \\
\hline \multicolumn{4}{|l|}{ Saxifragaceae } \\
\hline B. ciliata (Haw.) Sternb. & $\begin{array}{l}\text { Pathrkhar, sapdottar, } \\
\text { mehlu, shabla }\end{array}$ & H/W & Root paste (abscesses) \\
\hline \multicolumn{4}{|l|}{ Scrophulariaceae } \\
\hline B. crispa Benth. & Sandhyara & S/W & Leaf paste (skin burns and cuts) \\
\hline \multicolumn{4}{|l|}{ Smilacaceae } \\
\hline \multicolumn{4}{|l|}{ Solanaceae } \\
\hline C. annuum L. & Peepli & $\mathrm{H} / \mathrm{C}$ & $\begin{array}{l}\text { Leaf paste (skin burns) } \\
\text { Ripened fruit is fried in L. usitatissimum oil, pounded into paste (cuts) }\end{array}$ \\
\hline N. physalodes (L.) Gaertn. & Gheyien & S/W & $\begin{array}{l}\text { Root paste (abscesses) } \\
\text { Leaf paste (allergy) }\end{array}$ \\
\hline N. tabacum L. & Tambaku & $\mathrm{H} / \mathrm{W}$ & Warm leaves are applied with jaggery (abscesses) \\
\hline P. minima L. & Phophal-ghaien & H/W & $\begin{array}{l}\text { Fruit paste (skin burns, cuts, herpes zoster, problem of secretion from } \\
\text { scalp) }\end{array}$ \\
\hline S. aculeatissimum Jacq. & Kanteri & S/W & Fruit and seed paste (abscesses) \\
\hline S. americanum Mill. & Pattghaien & S/W & $\begin{array}{l}\text { Leaf paste (urticaria, cuts) } \\
\text { Paste is made by crushing leaves and fruits with } \\
\text { roots of } P \text {. benghalensis and black pepper (herpes zoster, skin } \\
\text { diseases) } \\
\text { Whole plant paste (skin burns) } \\
\text { Whole plant is crushed (abscesses) }\end{array}$ \\
\hline S. melongena L. & Baingan & $\mathrm{S} / \mathrm{C}$ & Stem ash (abscesses) \\
\hline S. tuberosum L. & Aalu & $\mathrm{H} / \mathrm{C}$ & Tuber is pounded (skin burns) \\
\hline
\end{tabular}


Table 1: (Continued)

\begin{tabular}{|c|c|c|c|}
\hline Families/botanical name of plants & Local name & $\mathrm{Ht} / \mathrm{Sc}$ & Part used and preparation (application/s) \\
\hline \multicolumn{4}{|l|}{ Urticaceae } \\
\hline B. macrophylla Hornem. & Chamrala, kalotra & $\mathrm{H} / \mathrm{W}$ & $\begin{array}{l}\text { Juice of young leaves (skin burns, cuts) } \\
8 \text {-10 leaves are pounded into paste with 2-3 black pepper (herpes zoster) } \\
\text { Leaf paste (abscesses) }\end{array}$ \\
\hline G. hirta (Blume ex Hassk.) Miq. & Kurand & H/W & $\begin{array}{l}\text { Root paste (abscesses) } \\
\text { Leaves are pounded with seeds of L. usitatissimum and jaggery into } \\
\text { paste (abscesses) }\end{array}$ \\
\hline U. dioica L. & Ainn & $\mathrm{H} / \mathrm{W}$ & $\begin{array}{l}\text { Leaf paste (itching) } \\
\text { Leaves are eaten as vegetable or chutney (allergy) }\end{array}$ \\
\hline \multicolumn{4}{|r|}{ ( } \\
\hline V. pilosa Blume & Banafsha, phali & H/W & $\begin{array}{l}\text { Roots and leaves paste (cuts) } \\
\text { Leaf paste (abscesses) } \\
\text { Whole plant paste (pimples) }\end{array}$ \\
\hline Vitaceae & & & \\
\hline $\begin{array}{l}\text { P. semicordata (Wall.) Planch. } \\
\text { Xanthorrhoeaceae }\end{array}$ & Amru-ri-bail & $\mathrm{Cl} / \mathrm{W}$ & Stem bark paste (abscesses) \\
\hline A. vera (L.) Burm. f. & Kaware & $\mathrm{H} / \mathrm{C}$ & $\begin{array}{l}\text { Warm leaf gel (abscesses) } \\
\text { Gel (Remove dark scars, skin burns, dandruff) }\end{array}$ \\
\hline \multicolumn{4}{|l|}{ Zingiberaceae } \\
\hline C. angustifolia Roxb. & & $\mathrm{H} / \mathrm{W}$ & Rhizome paste (abscesses) \\
\hline $\begin{array}{l}\text { C. longa L. } \\
\text { H. spicatum Sm. }\end{array}$ & $\begin{array}{l}\text { Haldi } \\
\text { Shati, banhaldi, shya, } \\
\text { shroli }\end{array}$ & $\begin{array}{l}\mathrm{H} / \mathrm{C} \\
\mathrm{H} / \mathrm{W}\end{array}$ & $\begin{array}{l}\text { Rhizome powder (cuts) } \\
\text { Rhizome paste (abscesses) }\end{array}$ \\
\hline
\end{tabular}

Ht/Sc: Habit/Source, H: Herb, S: Shrub, T: Tree, Cl: Climber, L: Liana, W: Wild, C: Cultivated, M: Market, W/C: Wild/Cultivated, D. bupleuroides: Dicliptera bupleuroides, J. adhatoda: Justicia adhatoda, A. aspera: Achyranthes aspera, A. bidentata: Achyranthes bidentata, C. tomentosa: Cyathula tomentosa, D. amaranthoides: Deeringia amaranthoides, A. cepa: Allium cepa, Z. carinata: Zephyranthes carinata, M. indica: Mangifera indica, C. asiatica: Centella asiatica, C. sativum: Coriandrum sativum, C. procera: Calotropis procera, C. spinarum: Carissa spinarum, C. roseus: Catharanthus roseus, C. dubia: Cryptolepis dubia, A. tortuosum: Arisaema tortuosum, C. esculenta: Colocasia esculenta, C. nucifera: Cocos nucifera, A. racemosus: Asparagus racemosus, D. indica: Drimia indica, A. adenophora: Ageratina adenophora, A. conyzoides: Ageratum conyzoides, A. nilagirica: Artemisia nilagirica, A. vulgaris: Artemisia vulgaris, B. anthelmintica: Baccharoides anthelmintica, C. minima: Centipeda minima, E. prostrata: Eclipta prostrata, G. gossypina: Gerbera gossypina, T. erecta: Tagetes erecta, T. patula: Tagetes patula, T. campylodes: Taraxacum campylodes, T. procumbens: Tridax procumbens, I. balsamina: Impatiens balsamina, B. aristata: Berberis aristata, O. indicum: Oroxylum indicum, B. ceiba: Bombax ceiba, C. zeylanicum: Cynoglossum zeylanicum, B. rapa: Brassica rapa, E. vesicaria: Eruca vesicaria, C. sativa: Cannabis sativa, V. jatamansi: Valeriana jatamansi, C. papaya: Carica papaya, T. arjuna: Terminalia arjuna, T. chebula: Terminalia chebula, C. reflexa: Cuscuta reflexa, I. nil: Ipomoea nil, B. pinnatum: Bryophyllum pinnatum, S. glaucophyllum: Sedum glaucophyllum, C. sativus: Cucumis sativus, L. siceraria: Lagenaria siceraria, D. deltoidea: Dioscorea deltoidea, L. ovalifolia: Lyonia ovalifolia, R. campanulatum: Rhododendron campanulatum, E. helioscopia: Euphorbia helioscopia, E. heterophylla: Euphorbia heterophylla, E. hirta: Euphorbia hirta, E. royleana: Euphorbia royleana, F. insignis: Falconeria insignis, J. curcas: Jatropha curcas, M. philippensis: Mallotus philippensis, M. pruriens: Mucuna pruriens, R. communis: Ricinus communis, A. precatorius: Abrus precatorius, A. gageana: Acacia gageana, B. variegata: Bauhinia variegata, B. monosperma: Butea monosperma, C. fistula: Cassia fistula, H. rosa-sinensis: Hibiscus rosa-sinensis, C. corylifolium: Cullen corylifolium, I. heterantha: Indigofera heterantha, L. culinaris: Lens culinaris, S. tora: Senna tora, V. mungo: Vigna mungo, Q. incana: Quercus incana, H. uralum: Hypericum uralum, J. regia: Juglans regia, A. parviflora: Ajuga parviflora, C. tomentosa: Callicarpa tomentosa, C. umbrosum: Clinopodium umbrosum, C. oppositifolia: Colebrookea oppositifolia, I. wightii: Isodon wightii, L. lanata: Leucas lanata, M. biflora: Micromeria biflora, P. mollis: Plectranthus mollis, P. benghalensis: Pogostemon benghalensis, P. mollissima: Premna mollissima, R. cinerea: Roylea cinerea, C. asiatica: Centella asiatica, A. parviflora: Ajuga parviflora, S. scandens: Scutellaria scandens, V. negundo: Vitex negundo, C. tamala: Cinnamomum tamala, L. usitatissimum: Linum usitatissimum, R. indica: Reinwardtia indica, L. inermis: Lawsonia inermis, P. granatum: Punica granatum, G. optiva: Grewia optiva, H. rosa-sinensis: Hibiscus rosa-sinensis, S. cordifolia: Sida cordifolia, T. govanianum: Trillium govanianum, A. indica: Azadirachta indica, M. azedarach: Melia azedarach, C. pareira: Cissampelos pareira, C. laurifolius: Cocculus laurifolius, S. glabra: Stephania glabra, F. auriculata: Ficus auriculata, F. benghalensis: Ficus benghalensis, F. carica: Ficus carica, F. palmata: Ficus palmata, F. racemosa: Ficus racemosa, F. religiosa: Ficus religiosa, M. serrata: Morus serrata, M. paradisiaca: Musa paradisiaca, S. aromaticum: Syzygium aromaticum, M. jalapa: Mirabilis jalapa, J. mesnyi: Jasminum mesnyi, A. multiflora: Aerides multiflora, O. corniculata: Oxalis corniculata, S. indicum: Sesamum indicum, P. emblica: Phyllanthus emblica, P. niruri: Phyllanthus niruri, P. tetraphylla: Peperomia tetraphylla, P. nigrum: Piper nigrum, P. depressa: Plantago depressa, P. zeylanica: Plumbago zeylanica, C. dactylon: Cynodon dactylon, D. falcatum: Drepanostachyum falcatum, E. coracana: Eleusine coracana, T. aestivum: Triticum aestivum, Z. mays: Zea mays, P. amplexicaulis: Persicaria amplexicaulis, P. capitata: Persicaria capitata, R. nepalensis: Rumex nepalensis, R. acris: Ranunculus acris, R. arvensis: Ranunculus arvensis, F. vesca: Fragaria vesca, P. supina: Potentilla supina, P. utilis: Prinsepia utilis, P. cerasoides: Prunus cerasoides, P. pashia: Pyrus pashia, R. ellipticus: Rubus ellipticus, C. spinosa: Catunaregam spinosa, G. aparine: Galium aparine, H. tetrasperma: Himalrandia tetrasperma, C. limon: Citrus limon, C. pseudolimon: Citrus pseudolimon, F. indica: Flacourtia indica, S. babylonica: Salix babylonica, A. indica: Aesculus indica, D. viscosa: Dodonaea viscosa, S. mukorossi: Sapindus mukorossi, B. ciliata: Bergenia ciliata, B. crispa: Buddleja crispa, S. aspera: Smilax aspera, C. annuum: Capsicum nnuun, N. physalodes: Nicandra physalodes, N. tabacum: Nicotiana tabacum, P. minima: Physalis minima, Physalis minima, S. aculeatissimum: Solanum aculeatissimum, S. americanum: Solanum americanum, S. melongena: Solanum melongena, S. tuberosum: Solanum tuberosum, B. macrophylla: Boehmeria macrophylla, G. hirta: Gonostegia hirta, U. dioica: Urtica dioica, V. pilosa: Viola pilosa, P. semicordata: Parthenocissus semicordata, A. vera: Aloe vera, C. angustifolia: Curcuma angustifolia, C. longa: Curcuma longa, H. spicatum: Hedychium spicatum 
Table 2: Families representing genera and species

\begin{tabular}{|c|c|c|}
\hline Families & $G^{*}$ & $S^{* *}$ \\
\hline Acanthaceae & 2 & 2 \\
\hline Amaranthaceae & 3 & 4 \\
\hline Amaryllidaceae & 2 & 2 \\
\hline Anacardiaceae & 1 & 1 \\
\hline Apiaceae & 2 & 2 \\
\hline Apocynaceae & 4 & 4 \\
\hline Araceae & 2 & 2 \\
\hline Arecaceae & 1 & 1 \\
\hline Asparagaceae & 2 & 2 \\
\hline Asteraceae & 10 & 12 \\
\hline Balsaminaceae & 1 & 1 \\
\hline Berberidaceae & 1 & 1 \\
\hline Bignoniaceae & 1 & 1 \\
\hline Bombacaceae & 1 & 1 \\
\hline Boraginaceae & 1 & 1 \\
\hline Brassicaceae & 2 & 2 \\
\hline Cannabaceae & 1 & 1 \\
\hline Caprifoliaceae & 1 & 1 \\
\hline Caricaceae & 1 & 1 \\
\hline Combretaceae & 1 & 2 \\
\hline Convolvulaceae & 2 & 2 \\
\hline Crassulaceae & 2 & 2 \\
\hline Cucurbitaceae & 3 & 3 \\
\hline Dioscoreaceae & 1 & 1 \\
\hline Ericaceae & 2 & 2 \\
\hline Euphorbiaceae & 5 & 8 \\
\hline Fabaceae & 10 & 10 \\
\hline Fagaceae & 1 & 1 \\
\hline Hypericaceae & 1 & 1 \\
\hline Juglandaceae & 1 & 1 \\
\hline Lamiaceae & 13 & 13 \\
\hline Lauraceae & 1 & 1 \\
\hline Linaceae & 2 & 2 \\
\hline Lythraceae & 2 & 2 \\
\hline Malvaceae & 3 & 3 \\
\hline Melanthiaceae & 1 & 1 \\
\hline Meliaceae & 2 & 2 \\
\hline Menispermaceae & 3 & 3 \\
\hline Moraceae & 2 & 7 \\
\hline Musaceae & 1 & 1 \\
\hline Myrtaceae & 1 & 1 \\
\hline Nyctaginaceae & 1 & 1 \\
\hline Oleaceae & 1 & 1 \\
\hline Orchidaceae & 1 & 1 \\
\hline 0xalidaceae & 1 & 1 \\
\hline Pedaliaceae & 1 & 1 \\
\hline Phyllanthaceae & 1 & 2 \\
\hline Piperaceae & 2 & 2 \\
\hline Plantaginaceae & 1 & 1 \\
\hline Plumbaginaceae & 1 & 1 \\
\hline Poaceae & 5 & 5 \\
\hline Polygonaceae & 2 & 3 \\
\hline Ranunculaceae & 1 & 2 \\
\hline Rosaceae & 6 & 6 \\
\hline Rubiaceae & 3 & 3 \\
\hline Rutaceae & 1 & 2 \\
\hline Salicaceae & 2 & 2 \\
\hline Sapindaceae & 3 & 3 \\
\hline Saxifragaceae & 1 & 1 \\
\hline Scrophulariaceae & 1 & 1 \\
\hline Smilacaceae & 1 & 1 \\
\hline Solanaceae & 5 & 8 \\
\hline Urticaceae & 3 & 3 \\
\hline Violaceae & 1 & 1 \\
\hline Vitaceae & 1 & 1 \\
\hline Xanthorrhoeaceae & 1 & 1 \\
\hline Zingiberaceae & 2 & 3 \\
\hline
\end{tabular}

$G^{*}$ : Genera, $S^{* *}$ : Species herpes zoster among which Physalis minima and Solanum americanum were most commonly used. Himalrandia tetrasperma was equally important for the same purpose but less known to people. The fungal infection of toes was cured using 19 species amongst which A. parviflora, Curcuma longa, Cynoglossum zeylanicum, Isodon wightii, Juglans regia, Pyrus pashia, Scutellaria scandens, and Terminalia chebula were common.

Plant or plant parts were used in medicinal preparations as per formulation, individually or in mixture forms. According to Saikia et al. (2006), the plant species which were used singly must be of great interest for discoveries of new drugs. Leaves were used most frequently (45.23\%) then roots $(12.50 \%)$, fruits and seeds $(11.90 \%$ each), latex $(8.33 \%)$, stem/rhizome/tuber (7.14\%), etc. (Figure 3). Leaves were also the most frequently used plant part in some previous ethnobotanical studies (Egharevba and Ikhatva, 2008; Rahman, 2013; Jatav and Mehta, 2013). According to Wet et al. (2013), the use of leaves is helpful in sustainable development.

A species of multiple use require extra attention toward their conservation. It has been observed that same parts of certain species have been used to treat different skin diseases such as Acacia gageana, Aerides multiflora, Centella asiatica, Colebrookea oppositifolia, Fragaria vesca, Sedum glaucophyllum, Tridax procumbens etc. However, in some cases, different parts of single species were used against same skin related ailment such as Cocculus laurifolius, P. pashia, Ranunculus acris, Terminalia arjuna etc. Traditional remedies were prepared using suitable methods. The majority of the remedies were paste (108), juice (28), crush (24), etc. Powder, decoction, roasted forms were also taken (Figure 4). These preparations were taken with other additives such as butter, clarified butter (ghee), curd, black pepper, honey, jaggery, and water. Black pepper was the most commonly used additive. These may either be added to increase the effect of medicines or to reduce the toxicity in plants if any. This study suggests that respondents have sound ethnobotanical knowledge and they are passing it on to the next generations also.

\section{CONCLUSION}

A total of 168 species have been recorded for the treatment of dermatological problems. The information collected from respondents indicates that they are aware about medicinal plants and their uses. This knowledge was inherited from their ancestors but vanishing gradually 


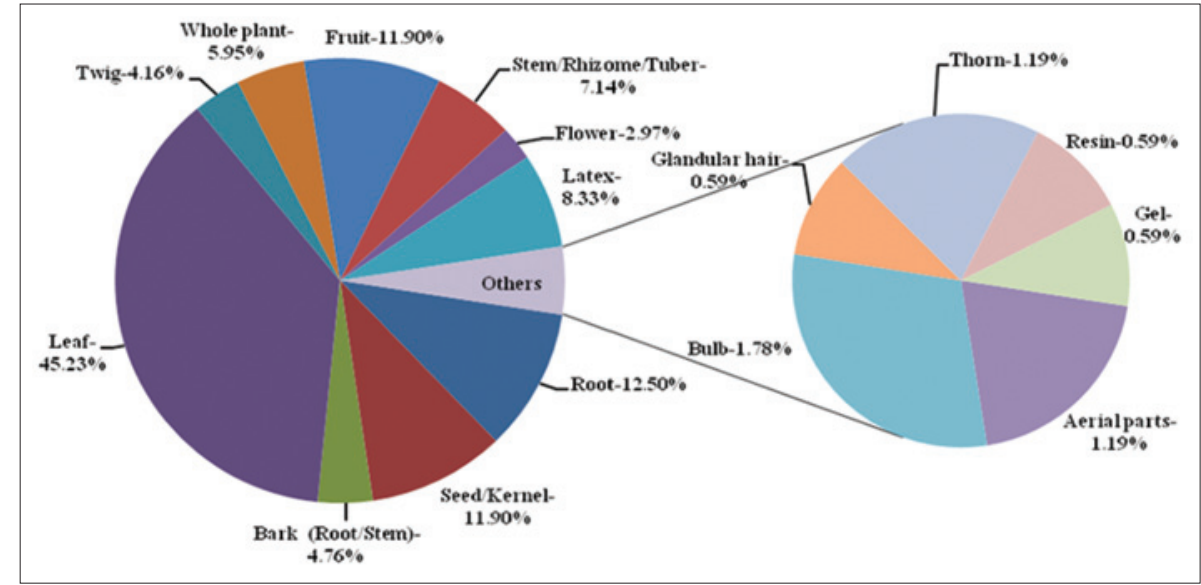

Figure 3: Various plant parts used in medicinal formulations

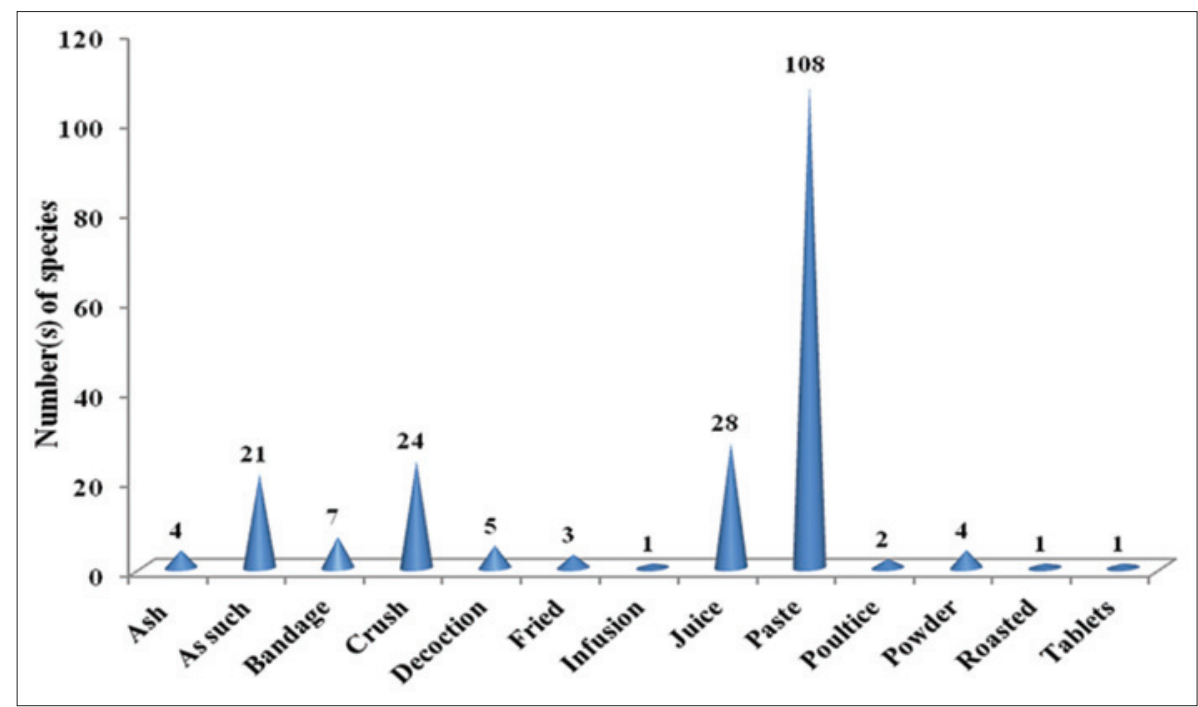

Figure 4: Various remedial preparations

and requires attention. Most of the plants were wild and herbs, so their conservation is necessary for utilization of generations to come. This can be done by encouraging local people for the cultivation of these plants. Furthermore, this preliminary study may act as a baseline for the discovery of new plant-based medicines. These plant species can be Studied in detail for different phytochemicals to understand their medicinal activity.

\section{ACKNOWLEDGMENTS}

The authors are grateful to Chairperson, Department of Botany, Panjab University, Chandigarh for providing necessary facilities during the study and University Grant Commission, New Delhi for providing UGC-BSR fellowship for research. We are also thankful to the respondents of the study area for their cooperation in the documentation of this valuable knowledge.

\section{REFERENCES}

Abbasi AM, Khan MA, Ahmad M, Zafar M, Jahan S, Sultana S. Ethnopharmacological application of medicinal plants to cure skin diseases and in folk cosmetics among the tribal communities of North-West Frontier Province, Pakistan. J Ethnopharmacol 2010;128:322-35.

Akharaiyi FC, Boboye B. Antibacterial and phytochemical evaluation of three medicinal plants. J Nat Prod 2010;3:27-34.

Central Ground Water Board. Ground Water Information Booklet. Mandi District, Himachal Pradesh: Government of India, Ministry of Water Resources; 2013.

Egharevba RK, Ikhatva MI. Ethno-medical uses of plants in the treatment of various skin diseases in Ovia North East, Edo state, Nigeria. Res J Agric Biol Sci 2008;4:58-64.

Govindasamy C, Arulpriya M. Antimicrobial activity of Acanthus ilicifolius: Skin infection pathogens. Asia Pac J Trop Dis 
2013;3:180-3.

Jatav R, Mehta R. Study of medicinal plants used in dermatological problems with special reference to Sahariya tribe of Shivpuri district of Madhya Pradesh. Indian J Appl Res 2013;3:60-2.

Joshi AR, Joshi K. Ethnomedicinal plants used against skin diseases in some villages of Kali Gandaki, Bagmati and Tadi Likhu watersheds of Nepal. Ethnobot Leafl 2007; 11:235-46.

Lazarou J, Pomeranz BH, Corey PN. Incidence of adverse drug reactions in hospitalized patients: A meta-analysis of prospective studies. J Am Med Assoc 1998;279:1200-5.

Muthu C, Ayyanar M, Raja N, Ignacimuthu S. Medicinal plants used by traditional healers in Kancheepuram district of Tamil Nadu, India. J Ethnobiol Ethnomed 2006;2:43.

Njoroge GN, Bussmann RW. Ethnotherapeutic management of skin diseases among the Kikuyus of Central Kenya. J Ethnopharmacol 2007;111:303-7.

Rahman AH. Traditional medicinal plants used in the treatment of different skin diseases of Santals at Abdullahpur village under Akkelpur Upazilla of Joypurhat district, Bangladesh. Biomed Biotechnol 2013;1:17-20.

Saikia AP, Ryakala VK, Sharma P, Goswami P, Bora U. Ethnobotany of medicinal plants used by Assamese people for various skin ailments and cosmetics. J Ethnopharmacol
2006;106:149-57.

Sidhu MC, Thakur S. Documentation of antidiabetic medicinal plants in district Mandi of Himachal Pradesh (India). Int J PharmTech Res 2015;8:164-9.

Sirsawat T, Suvarnasingh A, Maneenoon K. Traditional medicinal plants notably used to treat skin disorders nearby Khao Luang mountain hills region, Nakhon si Thammarat, SouthernThailand. J Herbs Spices Med Plants 2016;22:35-56

Suresh M, Ayyanar M, Amalraj L, Mehalingam P. Ethnomedicinal plants used to treat skin diseases in Pothigai hills of Western ghats, Tirunelveli district, Tamil Nadu, India. J Biosci Res 2012;3:112-21.

Uniyal B, Shiva V. Traditional knowledge on medicinal plants among rural women of the Garhwal Himalaya, Uttaranchal. Indian J Tradit Knowl 2005;4:259-66.

Wet HD, Nciki S, Vuuren SF. Medicinal plants used for the treatment of various skin disorders by a rural community in Northern Maputaland, South Africa. J Ethnobiol Ethnomed 2013;9:51.

WhiteTJ, Arakelian A, Rho JP. Counting the costs of drug-related adverse events. Pharmacoeconomics 1999;15:445-58.

Yirga G. Assessment of traditional medicinal plants in Endrta District, South-Eastern Tigray, Northern Ethiopia. J Med Plants Res 2010;4:255-60. 